\title{
Effect of asperity damage on shear behavior of single fracture
}

\author{
Anil Misra * \\ Department of Civil Engineering, University of Missouri-Kansas City, 350H Flarsheim Hall, 5100 Rockhill Road, \\ Kansas City, MO 64110, USA
}

Received 22 March 2002; accepted 16 April 2002

\begin{abstract}
Experimental studies have shown that fractures often exhibit shear resistance softening and dilatation under shearing loads. From a mechanistic viewpoint, these phenomena are a consequence of the fracture surface roughness and the material mechanical properties. Consequently, this paper utilizes a micromechanical model of fractures that explicitly considers asperity interactions on fracture surfaces. Elastic deformations and inelastic frictional sliding are considered at inclined asperity contacts. A modified spherical harmonic expansion is used to model the orientation distribution of asperity contacts. Evolution laws for asperity heights and asperity contact orientations are introduced to account for the change in surface roughness resulting from asperity damage under shear. Results obtained from the model show that the asperity contact orientation evolution law is essential for correctly modeling the softening and dilatation behavior of fractures. The model results are compared with experimental data culled from the literature.
\end{abstract}

(c) 2002 Elsevier Science Ltd. All rights reserved.

Keywords: Fracture; Damage mechanics; Rock; Shear behavior; Friction

\section{Introduction}

The shear behavior of fractures is of significance to the study of fracture mechanics problems, such as modeling of material damage, fracture propagation under shear and compression, and mechanics of jointed rock masses. Fracture shear behavior is, generally, complex and influenced by a variety of factors, including, among others, fracture surface roughness, material mechanical properties, and intrinsic friction. A number of efforts have been made to model the shear behavior using phenomenological and empirical approaches (see for example Refs. [1-3]). However, mechanistic approaches, that explicitly model surface topography and incorporate material mechanical properties and intrinsic friction, are expected to provide better insight into fracture friction behavior. For instance, it is well recognized that because of the surface roughness, the contact between fracture surfaces is through asperities and, consequently, the actual contact area is much smaller than the area of fracture surface [4,5]. Along the lines of mechanistic approach, the mechanical behavior of contacting surfaces has been studied by explicitly modeling the behavior of asperity

\footnotetext{
Tel.: +1-816-235-1285; fax: +1-816-235-1260.

E-mail address: misraa@umkc.edu (A. Misra).
} 
contacts (cf. [6,7]). Considering elastic deformation of asperity contacts and gaussian distributions of asperity heights, models of the normal and shear behavior for contacting rough surfaces have been derived [812]. Sliding at asperity contacts has also been considered to obtain friction behavior of contacting rough surfaces [13,14]. A review of some of these mechanistic/micromechanical methods is given in Ref. [15].

More recently, the author has developed a kinematically driven micromechanical methodology for contacting rough surfaces [16]. This methodology uses a directional distribution function of asperity contact orientations as an additional measure of surface roughness and was successfully used to derive a micromechanical model to replicate the anisotropic shear behavior of rock fractures [17]. In the present paper, we extend the micromechanical model to account for the effects of asperity damage on the fracture shear behavior. To this end, we introduce evolution laws for asperity heights and asperity contact orientations that account for the change in surface roughness resulting from asperity damage. The resulting micromechanical model is used to: (1) model the softening of fracture shear resistance under shear loading, (2) model the fracture shear strength, and (3) replicate the fracture dilatation behavior under shear loading. The model development, in this paper, is motivated by the experimentally observed shear resistance softening and dilatation behavior exhibited by single fractures [18,19].

In the following discussion, we first present a brief description of the micromechanical modeling methodology for obtaining fracture stress-deformation behavior. The evolution laws of asperity heights and asperity contact orientations are then developed utilizing experimental evidence culled from literature. The behavior of single fractures under shear loading calculated using the derived model is compared with experimental data from the literature. The micromechanical model is also used to perform limited parametric study of fracture shear behavior.

\section{Brief description of fracture micromechanical model}

We consider the micromechanical methodology wherein the stress-deformation behavior of a fracture is obtained by considering the force-deformation behavior of the asperity contacts and the statistical description of fracture surface topography [17]. At the asperity contact level, a local force-deformation relationship is defined that accounts for the elastic deformation and inelastic sliding at the contact. The stress-deformation relationship for a fracture is then derived utilizing the distribution functions of asperity heights and contact orientations, and the overall kinematic constraints and equilibrium conditions for the fracture. Here we give a brief description of the micromechanical model with the intent of demonstrating how the model is extended to include the effects of asperity damage.

\subsection{Force-deformation relationship for an asperity}

Considering both the elastic deformation and inelastic sliding, the relative motion, $\delta_{j}^{\mathrm{c}}$, at an asperity contact, may be decomposed into an elastic part, $\delta_{j}^{\mathrm{ce}}$, and an inelastic part, $\delta_{j}^{\mathrm{cp}}$, such that

$$
\delta_{j}^{\mathrm{c}}=\delta_{j}^{\mathrm{ce}}+\delta_{j}^{\mathrm{cp}}
$$

Subscripts follow the established tensor convention unless specified otherwise. The elastic deformations, $\delta_{j}^{\mathrm{ce}}$, at an asperity contact generate forces, $f_{i}^{c}$, which are related via the asperity contact stiffnesses, $K_{i j}^{\mathrm{c}}$, as follows:

$$
f_{i}^{\mathrm{c}}=K_{i j}^{\mathrm{c}} \delta_{j}^{\mathrm{ce}}
$$

We note that the asperity contact stiffnesses, $K_{i j}^{\mathrm{c}}$, generally depend upon the contact loading condition, such as the stiffness given by the Hertz-Mindlin contact theory [6]. We make no assumption regarding the form 
of this dependency in the derivation. As we will see later in the paper, both constant and non-constant asperity contact stiffnesses may be used for calculating a fracture behavior using the derived model.

The Amonton-Coulomb's friction law, expressed by the following inequality, governs the sliding at an asperity contact:

$$
f_{i}^{\mathrm{c}} q_{i}^{\mathrm{c}} \leqslant 0
$$

where

$$
q_{i}^{\mathrm{c}}=\zeta_{i}^{\mathrm{c}}+\mu n_{i}^{\mathrm{c}}
$$

$\zeta_{i}^{\mathrm{c}}$ is a unit vector in the sliding direction, $\mu$ is the asperity friction coefficient and $n_{i}^{\mathrm{c}}$ is a unit vector outwardly normal to the asperity contact. Combining Eqs. (1)-(4), the following relationship between the force and relative motion at a sliding asperity contact may be derived:

$$
f_{i}^{\mathrm{c}}=K_{i r}^{\mathrm{c}}\left(\delta_{r j}-M^{\mathrm{c}} K_{s j}^{\mathrm{c}} q_{\mathrm{s}}^{\mathrm{c}} \zeta_{r}^{\mathrm{c}}\right) \delta_{j}^{\mathrm{c}}
$$

where $\delta_{r j}(=1$ for $r=j ;=0$ for $r \neq j)$ is the Kronecker delta and the scalar $M^{\mathrm{c}}$ is given by

$$
M^{\mathrm{c}}=\left(K_{i j}^{\mathrm{c}} \zeta_{j}^{\mathrm{c}} q_{i}^{\mathrm{c}}\right)^{-1}
$$

\subsection{Number and orientations of asperity contacts}

The geometry of a fracture surface determines the orientations and the number of asperity contacts under a given loading condition. The composite topography of contacting fracture surfaces described via statistics of asperity contact heights, curvatures, and orientations may be utilized for this purpose [17,2023]. In general, these statistics are not independent of each other, and therefore their distribution functions may not be formulated independently. Nevertheless, the derivation of stress-deformation relationship is considerably simplified by considering these parameters independently. In the micromechanical model developed in this paper, the asperity contact curvatures are taken to be uniform.

The experimental measurements of topography suggests a skewed distribution for asperity heights, and several statistical distributions, such as chi-square distribution and gamma distribution have been used to model rough surfaces (see [12,23]). In this paper we use a gamma distribution for simplicity, such that the density function for asperity heights, $H(r)$, is expressed as

$$
H(r)=\frac{r^{\alpha} \mathrm{e}^{-r / \beta}}{\Gamma(\alpha+1) \beta^{\alpha+1}} \quad(0<r<\infty, \alpha>-1, \beta>0)
$$

where $\alpha$ and $\beta$ are parameters related to the mean and variance of the asperity heights as follows:

$$
\begin{array}{ll}
\text { mean : } & r_{m}=\beta(\alpha+1) \\
\text { variance : } & r_{\sigma}^{2}=\beta^{2}(\alpha+1)
\end{array}
$$

Parameter $\alpha$ is unitless while parameter $\beta$ takes the unit of asperity height. Fig. 1 illustrates the distribution of asperity heights for surfaces with varying roughness. Surfaces that have smaller average asperity height and narrow distributions of asperity heights are considered to be relatively smoother. Given that the total number of asperities per unit area of a fracture surface is $N$, then $N H(r) \mathrm{d} r$ denotes that number of asperity contacts in the interval represented by $r$ and $r+\mathrm{d} r$. It is convenient to define the asperity contact height with reference to the highest peak of the composite topography such that, asperity height, $r$, represents the overlap of the interacting surfaces. As a result, the total number of asperity contacts, under a given loading condition, is given by 


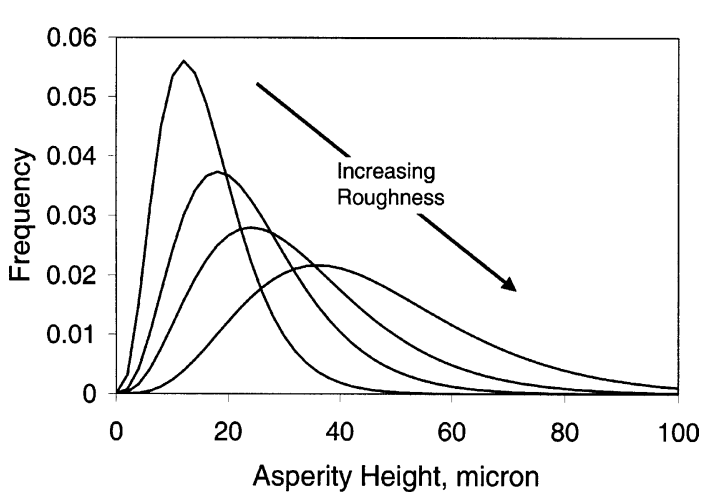

Fig. 1. Asperity height distributions with varying surface roughness.

$$
N_{r}=\int_{0}^{r} N H(r) \mathrm{d} r
$$

It is noteworthy, that the asperity contacts may be variously inclined and that the asperity contacts are not equally likely in all the orientations. We define an asperity contact orientation by considering the inclination of the asperity contact normal with respect to that of the fracture surface normal direction. As shown in Fig. 2, the orientation of an oblique asperity contact is defined by the azimuthal angle $\phi$ and the meridional angle $\theta$, measured with respect to a Cartesian coordinate system in which direction 1 is normal to the fracture surface. We use a modified form of spherical harmonics expansion to describe the concentrations of asperity contact orientations [17]. Considering the first two terms of spherical harmonics expansion in the domain: $0 \leqslant \theta \leqslant 2 \pi / a, 0 \leqslant \phi \leqslant 2$, the density function, $\xi(\Omega)$, of directional distribution of asperity contacts is expressed by

$$
\xi(\Omega)=\frac{a \sin a \theta}{2 \pi \sin \theta}\left[1+\frac{b}{4}(3 \cos 2 a \theta+1)+3 c \sin ^{2} a \theta \cos 2 \phi\right] \quad\left(0 \leqslant \theta \leqslant \frac{\pi}{2 a} ; 0 \leqslant \phi \leqslant 2 \pi ; a \geqslant 1\right)
$$

where angles $\phi$ and $\theta$ are defined in Fig. 2, and $\Omega$ represents the solid angle formed by $\phi$ and $\theta$. Parameters $a, b$, and $c$ determine the shape of the density function $\xi(\Omega)$. Thus, the product $N_{r} \xi(\Omega) \mathrm{d} \Omega$ denotes the number of asperity contacts $N_{\Omega}$ in the interval represented by solid angles $\Omega$ and $\Omega+\mathrm{d} \Omega$, that is

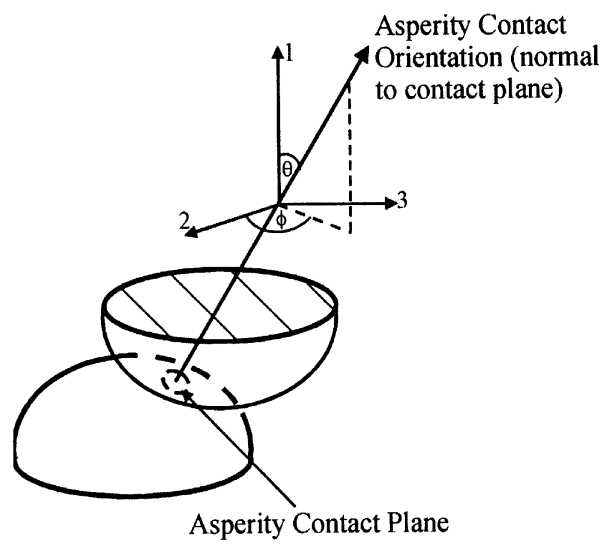

Fig. 2. Asperity contact orientation. 


$$
N_{\Omega}=N_{r} \xi(\Omega) \mathrm{d} \Omega
$$

The mean and variance of asperity contact orientations in the meridional direction may be obtained as the expectations $E[\theta]=\int \theta \xi(\Omega) \mathrm{d} \Omega$ and $E\left[(\theta-E[\theta])^{2}\right]=\int(\theta-E[\theta])^{2} \xi(\Omega) \mathrm{d} \Omega$, given by

$$
E[\theta]=\frac{6-b}{6 a} \text { and } E\left[(\theta-E[\theta])^{2}\right]=\frac{36 \pi-108+20 b-6 b \pi-b^{2}}{36 a^{2}}
$$

Similarly, the mean and variance of asperity contact orientations in the azimuthal direction may be obtained as the expectations $E[\phi]=\int \phi \xi(\Omega) \mathrm{d} \Omega$ and $E\left[(\phi-E[\phi])^{2}\right]=\int(\phi-E[\phi])^{2} \xi(\Omega) \mathrm{d} \Omega$, given by

$$
E[\phi]=\pi \quad \text { and } \quad E\left[(\phi-E[\phi])^{2}\right]=c+\frac{\pi^{2}}{3}
$$

Further, to ensure that the density function, $\xi(\Omega)$, is positive semi-definite, i.e. $\xi(\Omega) \geqslant 0$, the values of parameters $b$ and $c$ are bounded as follows:

$$
-1 \leqslant b \leqslant 2 \text { and }-\frac{1}{3}+\frac{b}{6} \leqslant c \leqslant \frac{1}{3}-\frac{b}{6}
$$

The density function in Eq. (10) has the ability to model surfaces with varying roughness. As illustrated in Fig. 3, the asperity contacts for smooth surfaces have a greater tendency to concentrate in the direction normal to the fracture than that for rough surfaces. It is noteworthy that, as parameter $a$ increases, the contact distribution concentrates towards the direction normal to the interface. In particular, the density function, $\xi(\Omega)$, behaves like a delta function in the limit $a \rightarrow \infty$ and yields an expectation $E[\theta]=0$, representing a concentrated contact orientation, normal to the interface of a perfectly smooth joint. Thus, parameter $a$, describes the extent of the asperity contacts in the meridional direction. Parameter, $b$, on the other hand, describes the shape of the contact distributions within the meridional extent of asperity contacts. For example, the extent of asperity contact inclination in meridional direction is $\pi / 2$ for $a=1$ and $\pi / 4$ for $a=2$, while, the shapes of contact distributions vary with the values of parameter $b$. Parameter $b=-1$, represents an interface on which the asperity contacts tend to orient closer to the horizon, i.e. $\theta=\pi / 2$. In contrast, parameter $b=2$, represents an interface on which preferred orientation is closer to the interface normal, i.e. $\theta=0$. For $b=0$, the asperity contacts are equally distributed in the meridional direction. Parameter, $c$, describes the shape of the contact distributions in the azimuthal direction and is useful for modeling the directional nature of fracture surface roughness.

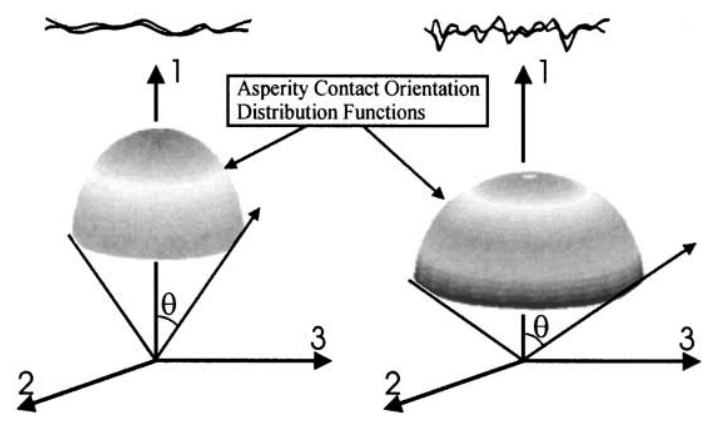

Fig. 3. Schematic depiction of asperity contact orientation distribution functions for smooth and rough interfaces. 
Considering the equilibrium of forces at a fracture surface, the overall traction $F_{i}$ on the fracture is obtained from the summation of the forces, $f_{i}^{\mathrm{c}}$, developed at asperities, which for a large number of asperity contacts may be written as the following integral equation:

$$
F_{i}=N \int_{r} \int_{\Omega} f_{i}^{\mathrm{c}} \xi(\Omega) H(r) \mathrm{d} \Omega \mathrm{d} r
$$

where traction $F_{i}$ is given as force per unit area, since $N$ is measured per unit area of a fracture. Under a given loading condition, an asperity contact may be sliding, separated or in elastic contact. Appropriately accounting for the asperity contact forces, and adopting the kinematic assumption that relative motion at an asperity, $\delta_{j}^{\mathrm{c}}$, is same as the relative motion of the interface, $\delta_{j}$, the relationship between the overall traction $F_{i}$ and the relative motion $\delta_{j}$ may be written as:

$$
F_{i}=C_{i j} \delta_{j}=\left[C_{i j}^{\mathrm{e}}-C_{i j}^{\mathrm{p}}\right] \delta_{j}
$$

where the superscripts e and $\mathrm{p}$ refer to the elastic and inelastic part of the fracture stiffness tensor, $C_{i j}$.

The elastic part, $C_{i j}^{\mathrm{e}}$, independent of the asperity contact sliding or separation, is given by

$$
C_{i j}^{\mathrm{e}}=N \int_{r} \int_{\Omega} K_{i j}^{\mathrm{c}} \xi(\Omega) H(r) \mathrm{d} \Omega \mathrm{d} r
$$

where the integration is performed over all the asperity contacts specified by the distributions of asperity contact orientations and asperity heights under a given interface normal load. On the other hand, the inelastic part depends critically upon the asperity contact loading condition. During an arbitrary shear loading, the inelastic part has two contributions: (1) from asperity contact sliding and (2) from separation of asperity contacts. It is expected that some of the oblique asperity contacts that are in contact under an initially applied normal load undergo unloading as the fracture is sheared. These contacts could potentially separate during the shear loading. Accounting for the two mechanisms of inelastic deformation, the inelastic part of the fracture stiffness tensor, $C_{i j}^{\mathrm{p}}$, is given by

$$
C_{i j}^{\mathrm{p}}=N \int_{r_{\mathrm{s}}} \int_{\Omega_{\mathrm{s}}} M^{\mathrm{c}} K_{i k}^{\mathrm{c}} \zeta_{k}^{\mathrm{c}} K_{n j}^{\mathrm{c}} q_{m}^{\mathrm{c}} \xi(\Omega) H(r) \mathrm{d} \Omega \mathrm{d} r+N \int_{r_{\mathrm{d}}} \int_{\Omega_{\mathrm{d}}} K_{i j}^{\mathrm{c}} \xi(\Omega) H(r) \mathrm{d} \Omega \mathrm{d} r
$$

where the integration is performed over the domain of sliding asperity contacts denoted by superscript $\mathrm{s}$ and separated asperity contacts denoted by superscript $\mathrm{d}$.

\section{Behavior of a single fracture under shear}

The domain of sliding and separated asperity contacts is not always known a priori, and only, under certain simple loading conditions and for constant asperity contact stiffness, the integrals in Eqs. (17) and (18) may be evaluated in closed forms $[16,17]$. For example, the sliding domain, under a normal deformation of a fracture is given by: $0 \leqslant \phi \leqslant 2 \pi$ and $\theta_{\mathrm{s}} \leqslant \theta \leqslant \pi / 2 a$, where $\theta_{\mathrm{s}}=\arctan (\lambda \mu)$, where $\lambda$ is the ratio of asperity stiffness in normal and tangential directions. For arbitrary shear loading conditions, such that $\delta_{1} \neq \delta_{2} \neq \delta_{3} \neq 0$, the sliding domain is not known a priori. Moreover, for non-constant asperity contact stiffness and changing surface roughness, the sliding domain evolves with loading. In addition, new asperity contacts are formed and existing contacts lost as the fracture is sheared. Numerically, the asperity separation may be detected by examining the total relative displacement in the normal direction of an asperity 
contact. Consequently, a secant fracture stress-displacement relationship is obtained by numerically integrating the following equations for each loading step:

$$
\begin{aligned}
& C_{i j}^{\mathrm{e}}=N \int_{0}^{r} \int_{0}^{2 \pi} \int_{0}^{\pi / 2 a} K_{i j}^{\mathrm{c}} \xi(\phi, \theta) \sin \theta \mathrm{d} \theta \mathrm{d} \phi H(r) \mathrm{d} r \\
& C_{i j}^{\mathrm{p}}=N \int_{0}^{r} \int_{\phi_{\mathrm{s}}} \int_{\theta_{\mathrm{s}}} M^{\mathrm{c}} K_{i k}^{\mathrm{c}} \zeta_{k}^{\mathrm{c}} K_{n j}^{\mathrm{c}} q_{m}^{\mathrm{c}} \xi(\phi, \theta) \sin \theta \mathrm{d} \theta \mathrm{d} \phi H(r) \mathrm{d} r+N \int_{0}^{r} \int_{\phi_{\mathrm{d}}} \int_{\theta_{\mathrm{d}}} K_{i j}^{\mathrm{c}} \xi(\phi, \theta) \sin \theta \mathrm{d} \theta \mathrm{d} \phi H(r) \mathrm{d} r
\end{aligned}
$$

where $\xi(\phi, \theta)$ is the asperity contact orientation distribution given by Eq. (10), $H(r)$ is asperity height distribution given by Eq. (7), $r=r_{0}+\delta_{1}$, and $r_{0}$ is the asperity overlap at $\delta_{1}=0$. Further, for shear loading under controlled normal stress $F_{1}$, an iterative procedure is required to obtain the contact forces and displacements. In the subsequent discussion we first discuss the form of non-constant asperity contact stiffness used in our analysis, we then develop evolution laws of asperity heights and asperity contact orientations and present comparison of model calculations and measured fracture shear behavior.

\subsection{Non-linear asperity contact stiffness}

It is convenient to express the asperity stiffness tensor, $K_{i j}^{\mathrm{c}}$, in terms of asperity stiffness that describes the behavior along the direction of normal and tangent to an asperity contact, such that

$$
K_{i j}^{\mathrm{c}}=K_{\mathrm{n}}^{\mathrm{c}} n_{i}^{\mathrm{c}} n_{j}^{\mathrm{c}}+K_{\mathrm{s}}^{\mathrm{c}}\left(s_{i}^{\mathrm{c}} s_{j}^{\mathrm{c}}+t_{i}^{\mathrm{c}} t_{j}^{\mathrm{c}}\right)
$$

where $K_{\mathrm{n}}$ and $K_{\mathrm{s}}$ denote asperity stiffness along the normal and tangential direction of the asperity. The unit vector $\mathbf{n}$ is normal to the asperity contact surface and vectors $\mathbf{s}$ and $\mathbf{t}$ are arbitrarily chosen on the plane tangential to the asperity contact surface, such that nst forms a local Cartesian coordinate system. It is noted that the stiffness term that cross-link normal and shear behavior are assumed to be negligible in accordance with the theories for contact of smooth non-conforming bodies. Furthermore, for modeling the fracture behavior under general loading conditions, non-linear asperity contact stiffness, that depend upon contact forces or displacements are preferable.

Considering the Hertz-Mindlin contact theory of perfectly smooth elastic surfaces as well as other theories of elasto-plastic interfaces (see Refs. [6,24]), the secant normal asperity stiffness, $K_{\mathrm{n}}=f_{\mathrm{n}} / \delta_{\mathrm{n}}$, may be taken to depend upon the normal asperity deformation, $\delta_{\mathrm{n}}$, according to the following power law:

$$
K_{\mathrm{n}}=\lambda K \delta_{\mathrm{n}}^{\eta}
$$

where $K, \lambda$ and $\eta$ are constants. The asperity stiffness, $K_{\mathrm{n}}$, given by Eq. (21), becomes identical with the Hertz-Mindlin stiffness for contact of perfectly smooth elastic spheres when

$$
\lambda=\frac{2-v}{2(1-v)}, \quad \eta=\frac{1}{2} \quad \text { and } \quad K=\frac{8 G \sqrt{R}}{3(2-v)}
$$

where $G$ is the shear modulus, $v$ is Poisson's ratio and $R$ is asperity radius of curvature. It is noteworthy that the exponent $\eta$ can vary from 0 for perfectly plastic to $1 / 2$ for perfectly elastic behavior at contact of perfectly smooth spherical asperities [6].

The tangential asperity stiffness, $K_{\mathrm{s}}$, has, in general, a complex dependence upon the asperity loading conditions in the tangential direction [25]. Since this paper focuses upon monotonic shear of fractures, we consider the case of constant normal asperity force and monotonically increasing asperity shear force. The following asperity force-displacement relationship has been derived for this loading condition, considering partial slip at contact edge with increasing contact shear displacement: 


$$
f_{\mathrm{s}}=\mu K_{\mathrm{n}} \delta_{\mathrm{n}}\left[1-\left(1-\frac{\delta_{\mathrm{s}}}{\mu \lambda \delta_{\mathrm{n}}}\right)^{3 / 2}\right]
$$

where $f_{\mathrm{s}}$ is the asperity shear force, and $\delta_{\mathrm{n}}$ and $\delta_{\mathrm{s}}$ are the asperity normal and shear displacements. It can be shown that in this case, the tangential asperity stiffness, $K_{\mathrm{s}}$, varies from $3 K_{\mathrm{n}} / 2 \lambda$, for vanishingly small asperity shear force, to $K_{\mathrm{n}} / \lambda$, for asperity shear force at incipient sliding condition. In this paper, we use the following truncated series representation for the tangential asperity stiffness, $K_{\mathrm{s}}=f_{\mathrm{s}} / \delta_{\mathrm{s}}$ :

$$
K_{\mathrm{s}}=\frac{3 K_{\mathrm{n}}}{2 \lambda}\left[1-\frac{1}{4 \mu \lambda} \frac{\delta_{\mathrm{s}}}{\delta_{\mathrm{n}}}-\frac{1}{24 \mu^{2} \lambda^{2}}\left(\frac{\delta_{\mathrm{s}}}{\delta_{\mathrm{n}}}\right)^{2}-\mathrm{O}\left(\frac{\delta_{\mathrm{s}}}{\delta_{\mathrm{n}}}\right)^{3}\right]
$$

which yields a variation from $3 K_{\mathrm{n}} / 2 \lambda$, for vanishingly small asperity shear force, to $1.063 K_{\mathrm{n}} / \lambda$, for asperity shear force at incipient sliding condition.

\subsection{Effect of asperity damage on fracture roughness}

During the shear deformation of a fracture, the interlocked asperities undergo damage under the complex stress conditions that develop at the asperity contacts. The asperity damage manifests as a change in roughness of the fracture surface. Fig. 4 shows the before and after asperity height distributions of a monotonically sheared fracture in Westerly granite sample. The solid dots in Fig. 4 denote the measured asperity height distribution based on data from Ref. [26]. The solid curves are the best-fit gamma distributions with following parameters: $\alpha=3$ and $\beta=8 \mu \mathrm{m}$ for fracture surface before shear, and $\alpha=7.5$ and $\beta=3.3 \mu \mathrm{m}$ for fracture surface after shear deformation of $1 \mathrm{~cm}$. The best-fit gamma distributions yield mean asperity heights of 32 and $28.05 \mu \mathrm{m}$ and asperity height standard deviations of 16 and $9.6 \mu \mathrm{m}$ before and after shear, respectively. The mean asperity height as well as the variance of the asperity height distribution becomes smaller with shear. Smaller mean and variance of asperity heights results from the wear of asperities to produce a smoother fracture surface with uniform asperity heights. Further, it is observed that the asperity height distribution after shear are truncated close to peaks, suggesting that the surface roughness evolves by progressive removal of the highest asperities from the surface. Clearly, the asperity height distribution has a relatively small change even after a shear deformation of $1 \mathrm{~cm}$. It is noteworthy that the shear deformations at yield for these Westerly granite fractures were, typically, measured to be less than $20 \mu \mathrm{m}[27,28]$. Consequently, little change in asperity height distribution may be expected for shear deformations close to or just beyond yield deformation.

Fig. 5 gives the asperity contact angle distribution for a rough and a smooth fracture surface obtained from the analysis of measured profiles [26]. As seen from Fig. 5, the smoother surface has a smaller mean

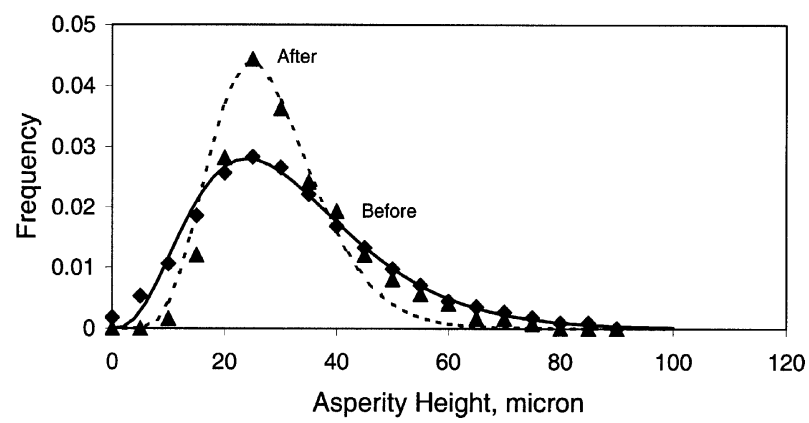

Fig. 4. Asperity height distributions before and after shear. 


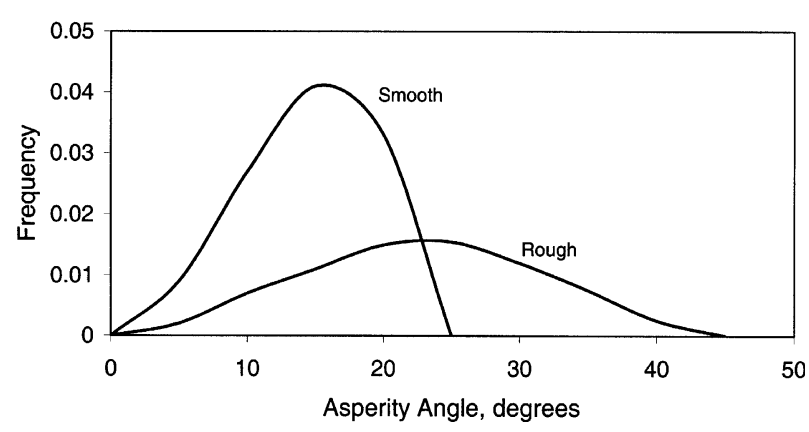

Fig. 5. Asperity orientation for smooth and rough fracture surface.

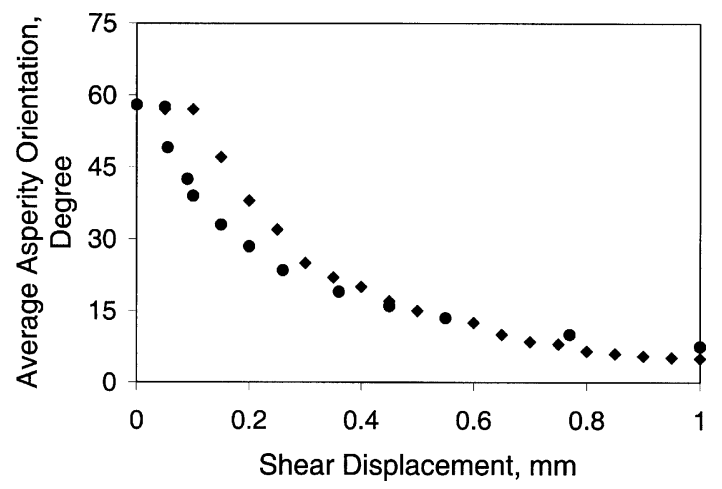

Fig. 6. Evolution of average asperity orientation with shear.

asperity angle as compared to rougher surface. Furthermore, asperity angle distribution for the smoother surface is narrower signifying a smaller variance of asperity angles. The data in Fig. 5 indicates that, as the surface becomes relatively smoother, the mean asperity angle and the variance of asperity angle distribution decrease. Fig. 6 gives the measured evolution of mean asperity contact orientation with shear deformation. The mean asperity contact orientation is found to decrement in a hyperbolic fashion with fracture shear. It is noteworthy that for a small initial shear displacement, the asperity contact orientation remains constant indicating that a critical shear displacement, $\delta_{c}$, is required before the asperity damage commences. The critical shear displacement has similarity with the critical interlocking distance required to shear off asperities discussed in Ref. [26] in the context of wear of contacting rough surfaces. The measured data depicted by solid symbols in Fig. 6 is obtained from the analysis of lab experiments on fractures in concrete presented in Ref. [19]. To the knowledge of the author, measured data describing the evolution of asperity contact orientation variance is not available in the literature.

\subsection{Asperity height and contact orientation evolution laws}

Based upon the experimental evidence of the effect of asperity damage on the statistical properties of a surface roughness, we postulate simple damage laws to describe the evolution of asperity height and asperity contact orientation distributions. In this regard we note that previous theoretical and laboratory studies on wear of contacting surfaces have related surface wear to shear displacement and shear work (cf. $[26,29])$. In this paper, we assume that the asperity damage is related to the work done to shear the fracture. 
Consequently, we propose that the standard deviation of asperity heights, $r_{\sigma}$, decrease in accordance with the following exponential law:

$$
r_{\sigma}=r_{\sigma 0} \exp \left(-k_{1} E_{\mathrm{s}}\right)
$$

where $r_{\sigma 0}$ is the standard deviation of asperity heights for shear displacement less than the critical shear displacement, $\delta_{\mathrm{c}} ; k_{1}$ is a material constant; and $E_{\mathrm{s}}$ is the total work done to shear the fracture. Given that the experimental evidence suggests only a small change in mean asperity heights during shear, we assume the mean asperity height to remain unchanged. As an example, Fig. 7 gives the variation of asperity height standard deviation with shear displacement for $k_{1}=0.01$. The associated evolution of asperity height distribution is shown in Fig. 8, which gives the initial height distribution as well as the height distributions after 2 and $5 \mathrm{~mm}$ shear displacements (depicted by points 1, 2 and 3 in Fig. 7). As seen from the asperity height distributions, the surface roughness evolves by progressive removal of the highest asperities of the initial surface and the gradual decrement of the asperity height variance. The initial surface is defined by the following gamma distribution parameters: $\alpha=4$ and $\beta=1.5 \mathrm{~mm}$, such that the asperity height mean and standard deviation are 7.5 and $3.35 \mathrm{~mm}$, respectively. The asperity height standard deviations for distributions 2 and 3 are 2.64 and $1.84 \mathrm{~mm}$, respectively.

We also propose that the mean asperity contact orientation parameter, $a$, increase linearly with the shear work as follows,

$$
a=a_{0}\left(1+k_{2} E_{\mathrm{s}}\right)
$$

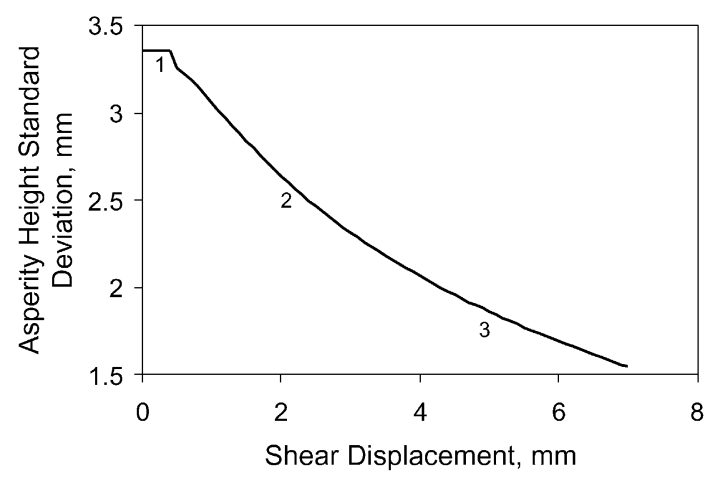

Fig. 7. Evolution law for asperity height standard deviation.

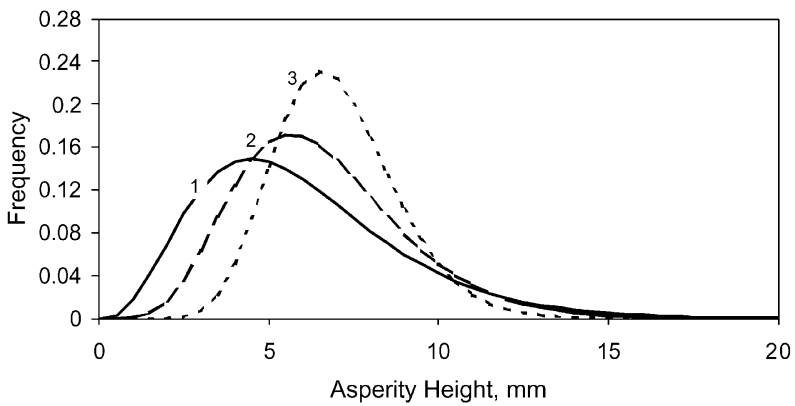

Fig. 8. Asperity height distributions showing the effect of evolution law given in Fig. 7. 


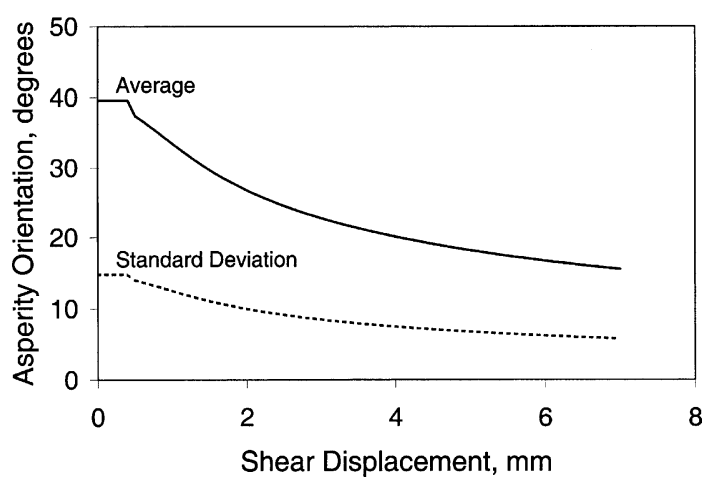

Fig. 9. Evolution of mean and standard deviation of asperity contact orientation.

where $a_{0}$ is the asperity contact orientation parameter for shear displacement less than the critical shear displacement, $\delta_{c}$; and $k_{2}$ is a material constant. Assuming, that asperity contact orientation parameter $b=0$, the mean and variance of asperity contact orientations in the meridional direction may be obtained from Eq. (12). Combining Eqs. (12) and (26), we see that the mean asperity orientation follows a hyperbolic decrement law. As an example, Fig. 9 gives the evolution of mean and standard deviation of asperity contact orientations in the meridional direction with shear deformation for $k_{2}=0.01$. As evident from the comparison of Figs. 6 and 9, the evolution law in Eq. (26) captures the essence of the evolution of asperity contact orientation with shear deformation.

\subsection{Fracture softening behavior under shear}

The asperity height and contact orientation evolution laws defined in the previous section are utilized with Eqs. (19a) and (19b) to obtain the shear behavior of fractures under constant normal stress. In Fig. 10, we compare the shear resistance-shear displacement curves obtained from the present model with the experimental data on three types of model fractures with different surface roughness reported by Bandis et al. [18]. Shear resistance is defined as the ratio $F_{2} / F_{1}$, where $F_{1}$ and $F_{2}$ are fracture normal and shear stresses, respectively. Solid lines give the calculated curves while the experimental data are indicated by symbols. The calculated curves were obtained using asperity friction coefficient of $\mu=0.85$, and the following stiffness parameters: $\eta=0.5, K=200 \mathrm{MPa} \mathrm{mm}^{1 / 2}$, and $\lambda=1.2$. These stiffness parameters are based upon the Hertz-Mindlin stiffness parameters given in Eq. (22) for a shear modulus $G=750 \mathrm{MPa}$, Poisson's ratio $v=0.3$, and asperity radius of curvature $R=30 \mu \mathrm{m}$. The shear modulus and asperity radius of curvature are based upon data given in Ref. [18]. In absence of measured data of surface topography we assume that $\sim 95 \%$ of the asperity are in contact under initial conditions, such that the initial overlap for the fracture is $r_{0}=10 \mathrm{~mm}$. The initial asperity height distributions and its evolution with shear are taken to be same for the three fracture types. The parameters for initial asperity height distributions and its evolution with shear are same as for the distributions shown in Fig. 8. The asperity contact density is taken to be $N=10$ per $\mathrm{cm}^{2}$ for the three fracture types. The variation in surface roughness are modeled by assuming the following asperity contact orientation parameter for the three fracture types: $a=4$ for smooth, $a=2.4$ for intermediate and $a=1.4$ for the rough fractures. For all the fractures the asperity contact orientation parameters $b$ and $c$ are assumed to be zero. The initial mean asperity orientation, calculated as the expectation $E(\theta)=\int \theta \xi(\Omega) \mathrm{d} \Omega$, is $14^{\circ}$ for smooth, $24^{\circ}$ for intermediate and $41^{\circ}$ the rough fractures. The evolution of asperity contact orientation parameter $a$ follows Eq. (26) with $k_{2}=0.015$, such that mean and standard deviation of asperity contact orientation vary in the manner similar to that in Fig. 9. 

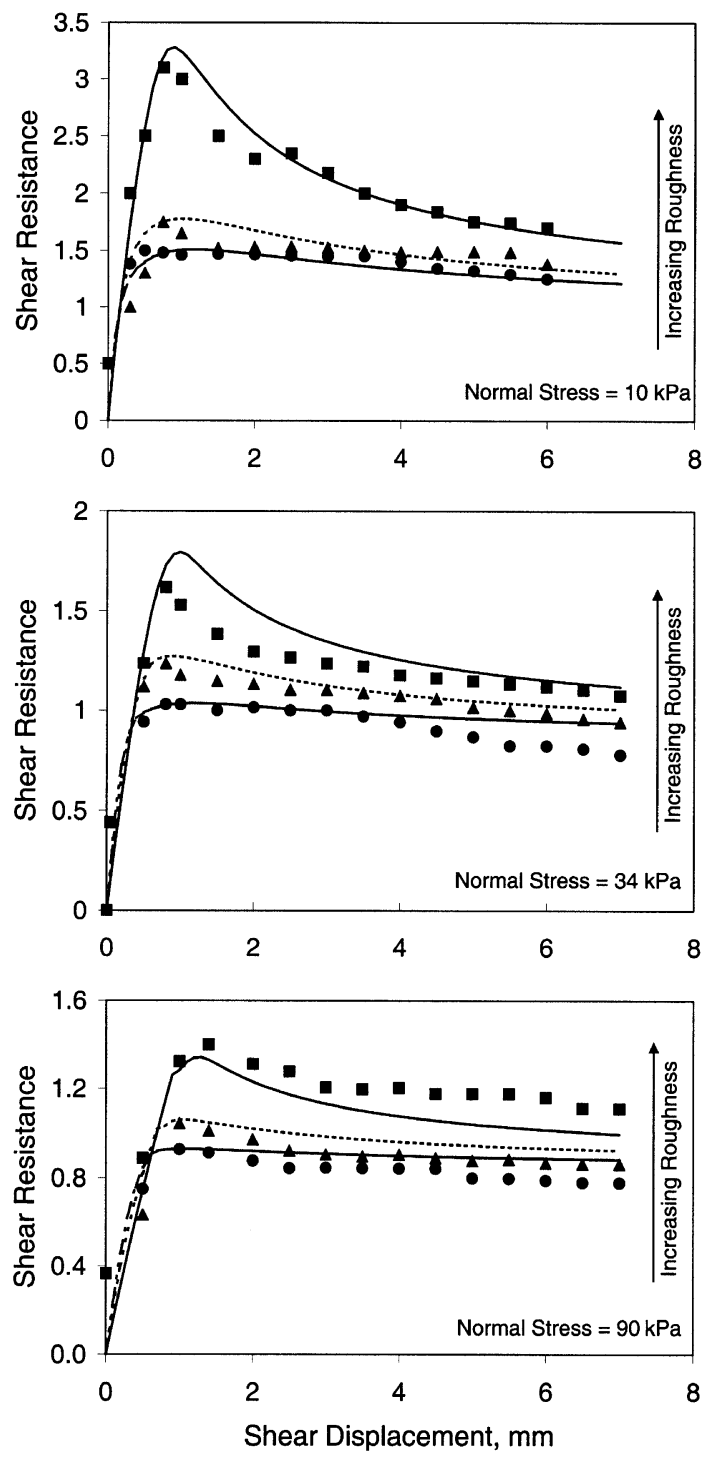

Fig. 10. Comparison of measured and calculated shear behavior of single fractures with different surface roughness under normal stresses of 10,34 , and $90 \mathrm{kPa}$, respectively.

As seen from Fig. 10, sheared fractures often exhibit a softening behavior past the peak shear strength. It is encouraging to note that the proposed asperity contact orientation evolution law in combination with the micromechanical model correctly replicates the softening behavior of fractures. Based upon model results, we observe that the softening behavior mainly depends upon (1) initial surface roughness, (2) rate and process of surface roughness evolution caused by asperity damage. The effect of initial surface roughness upon shear resistance versus shear displacement behavior may be seen from Fig. 10. The rough fracture has a considerably pronounced softening past peak shear resistance while the smooth fracture exhibits almost no softening behavior. 


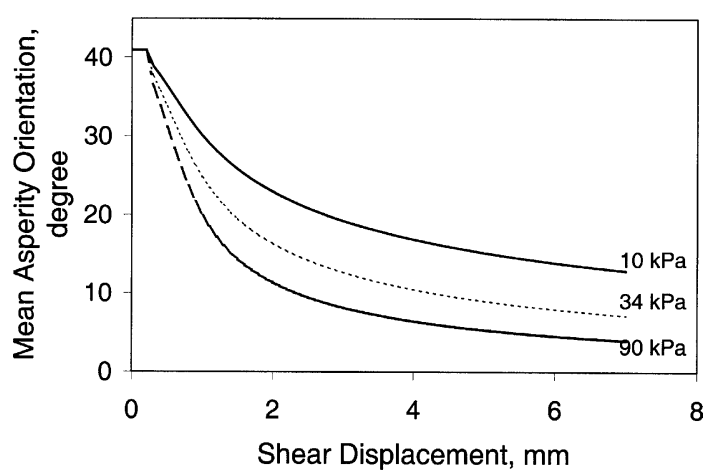

Fig. 11. Effect of fracture normal stress upon the evolution of mean asperity contact orientation.

Furthermore, for a given initial surface roughness and material properties, the asperity damage and the consequent change in roughness is critically affected by the fracture normal stress. Fig. 11 shows the effect of fracture normal stress upon the evolution of mean asperity contact orientation for the rough fracture. We observe that close to peak shear resistance, corresponding to shear displacements in the range of 1-2 $\mathrm{mm}$, larger shear stresses are required to achieve the same shear displacement under higher normal stresses. As a result, under higher normal stresses, the asperity damage occurs at a faster rate below peak shear resistance. Consequently, lower peak shear resistance is obtained under higher fracture normal stress, as exemplified in Fig. 12(a), which gives the peak shear resistance, expressed as friction angle $\tan ^{-1}\left(F_{2} / F_{1}\right)$, plotted against the fracture normal stress. The corresponding peak shear stress versus fracture normal stress

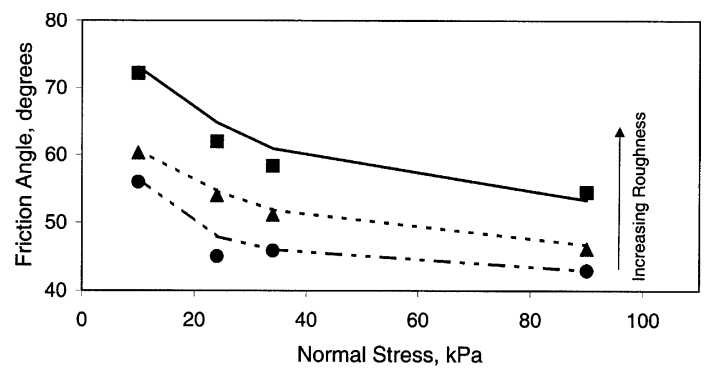

(a)

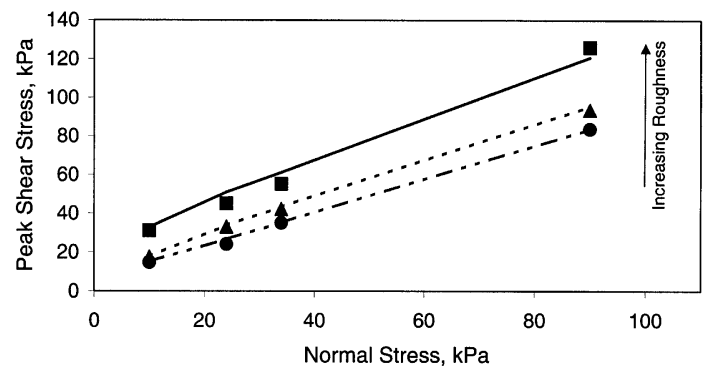

(b)

Fig. 12. Comparison of measured and calculated shear failure behavior of single fractures with different surface roughness: (a) friction angle, and (b) failure envelopes. 
behavior is given in Fig. 12(b). It is remarkable that the friction angles at higher stresses are considerably lower for a given initial surface roughness. Past peak, in the softening regime, the asperity damage rate is almost same under different normal stresses, therefore, the rate of softening is slower for higher fracture normal stresses as seen in Fig. 10.

\subsection{Fracture dilatation behavior under shear}

In Fig. 13(a), we compare fracture dilatation behavior calculated using the present model with the experimental data reported in Ref. [18] for the three types of fractures discussed in Section 3.4. The vertical displacements versus shear displacement curves are obtained for constant normal stress shearing of these fractures. Ref. [18] gives the measured data for a constant normal stress of $24 \mathrm{kPa}$ only. In Fig. 13(b), we show the effect of fracture normal stress upon the dilatation behavior. We observe that the fracture dilatation increases with roughness. We also observe that larger compression and lower dilatation is obtained at higher normal stresses. In addition, as the shear displacements increase, the vertical displacements tend

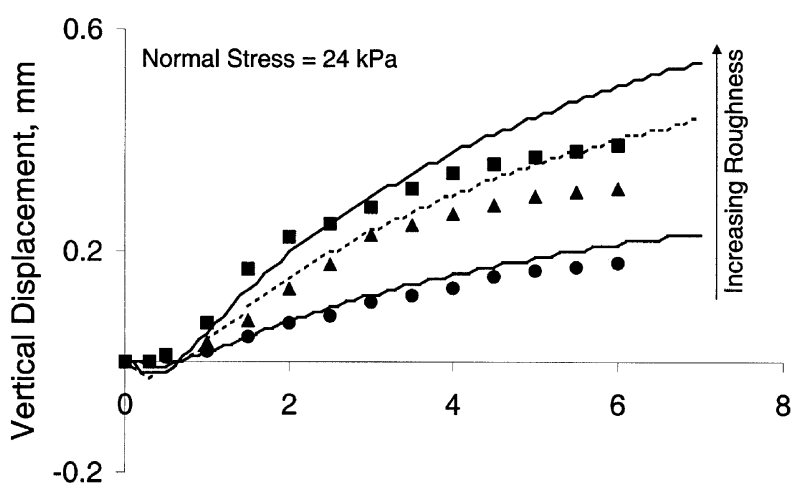

Shear Displacement, $\mathrm{mm}$

(a)

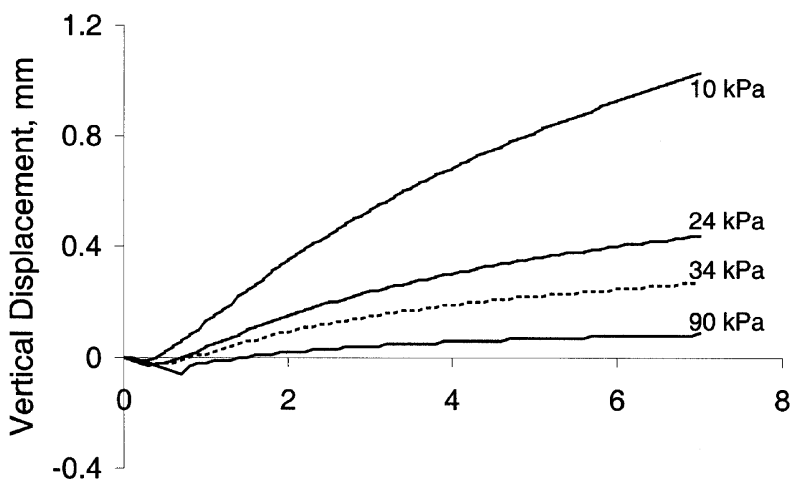

Shear Displacement, mm

(b)

Fig. 13. (a) Comparison of measured and calculated shear-dilatation behavior of single fractures with different surface roughness. (b) Effect of fracture normal stress on dilatation behavior. 
to a constant steady state value. The model correctly predicts the increased dilatation with increasing roughness. Notably, the model replicates the trends of dilatation behavior encouragingly well, given that the values of parameters used for model calculations are same as those used for Fig. 10, even though the fractures are not identical. We also note that the constant vertical displacement at large shear displacement results from the manner in which the surface roughness evolves with shear displacement. At large shear displacement, the asperity contact orientations also tend to a steady state condition as seen from the evolution law in Fig. 9.

The effect of fracture dilatation is further exemplified by studying the fracture shear behavior under a constant fracture opening condition. Under this type of loading condition, the fracture normal stress varies as the fracture is sheared. Figs. 14 and 15 give a comparison of calculated and measured stress-shear displacement curve for a fracture sheared under constant normal opening. Experimental data are indicated by symbols, while the solid line gives the calculated curves. The experimental data was obtained from Ref. [19] for constant crack width shear of fractures in cement concrete. The calculated curves were obtained using asperity friction coefficient of $\mu=0.6$, and the following stiffness parameters: $\eta=0.5, K=250 \mathrm{GPa}$ $\mu \mathrm{m}^{1 / 2}$, and $\lambda=1.2$. These stiffness parameters are based upon the Hertz-Mindlin stiffness parameters given in Eq. (22) for a shear modulus $G=22.5 \mathrm{GPa}$, Poisson's ratio $v=0.3$, and asperity radius of curvature $R=50 \mu \mathrm{m}$. The shear modulus is estimated as a typical value for cement concrete and the radius of curvature is assumed based upon a nominal particle size of $0.1 \mathrm{~mm}$. In absence of measured data of surface topography we assume that $\sim 10 \%$ of the asperity are in contact under initial conditions, such that the initial overlap for the fracture is $r_{0}=1.1 \mathrm{~mm}$. The initial asperity height distributions parameters are taken to be: $\alpha=8$ and $\beta=0.25 \mathrm{~mm}$, such that the mean and standard deviation of asperity heights are 2.25 and 0.56 $\mathrm{mm}$, respectively. The contact density is taken to be $N=1$ per $\mathrm{cm}^{2}$ and the asperity contact orientation parameter $a=2$, such that the initial mean asperity orientation is $29^{\circ}$. The evolution of asperity height standard deviation follows Eq. (25) with $k_{1}=0.001$, and the evolution of asperity contact orientation parameter $a$ follows Eq. (26) with $k_{2}=0.015$.

As seen from Fig. 14, the present model captures the shear strength and softening phenomena reasonably well. Furthermore, the model correctly replicates the variation of normal stress with shear displacement. The normal stress variation relates closely to the dilatation behavior of fractures. As seen from Fig. 15(a), the normal stress first decreases corresponding to the tendency of a fracture to close when the shear displacement is small. The normal stress then increases to a steady state as the fracture has a tendency to dilate to a steady state opening corresponding to the dilatation behavior shown in Fig. 13.

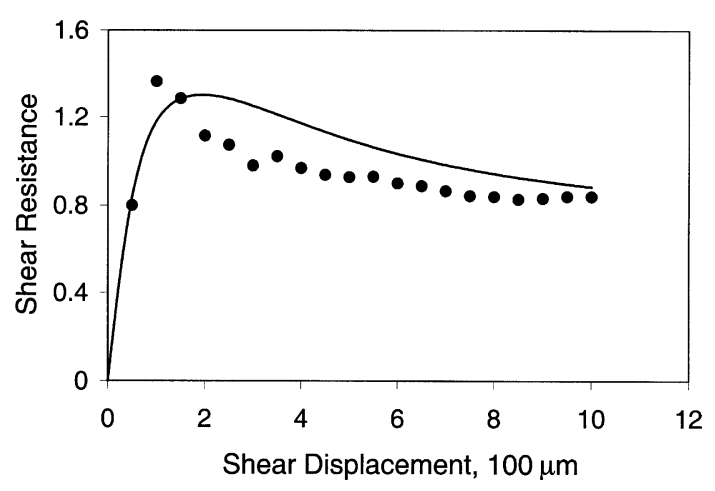

Fig. 14. Comparison of measured and calculated shear behavior of single fractures under constant normal fracture opening. 


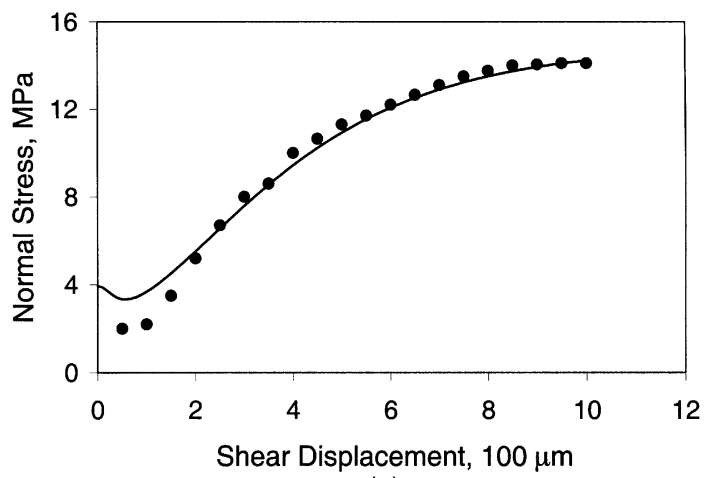

(a)

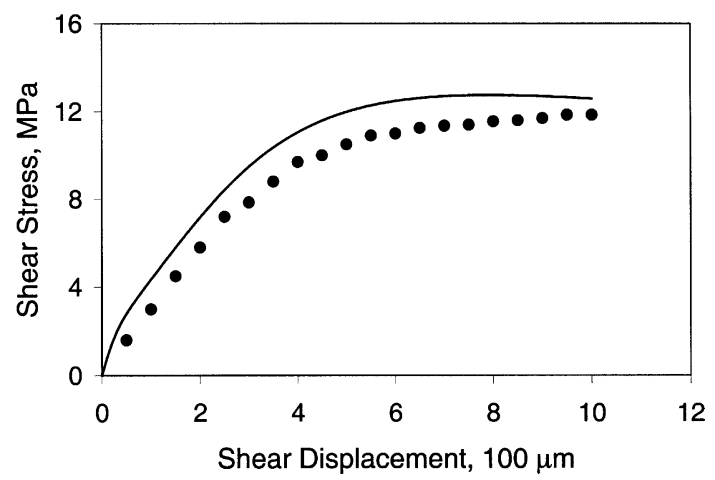

(b)

Fig. 15. Comparison of measured and calculated (a) normal stress, and (2) shear stress of a single fracture sheared under constant normal fracture opening.

\section{Concluding remarks}

A micromechanical model is developed to study the effect of asperity damage on fracture shear behavior. The aim of this effort is to: (1) investigate the mechanisms of fracture shear resistance softening under shear loading, (2) model the fracture shear strength, and (3) replicate the fracture dilatation behavior under shear loading. We recognize that the above three aspects of fracture behavior are critically influenced by interaction between asperities and the evolution of fracture surface roughness during shear. Consequently, we use a micromechanical methodology that explicitly considers asperity interactions and surface roughness, as characterized by statistical distributions of asperity heights, and asperity contact orientations. Gamma distribution is used for describing asperity heights, while a modified spherical harmonic expansion is used to model the asperity contact orientation distribution. The fracture surface roughness change as a result of asperity damage is modeled by using evolution laws for asperity height and asperity contact orientation distributions. Considering the previous studies on wear of contacting surfaces, we assume that the asperity damage and, consequently, the evolution laws are related to energy required to shear the fracture.

Experimental observation shows that the mean asperity height as well as the variance of the asperity height distribution becomes smaller with shear. Smaller mean and variance of asperity heights results from the wear of asperities to produce a smoother fracture surface with uniform asperity heights. Experimental evidence also suggests that the change in mean asperity heights is negligibly small at small shear 
displacements. Consequently, the evolution of asperity height distribution is modeled using an exponential decrement law for asperity height standard deviation. In addition, the mean and variance of asperity contact orientation also decrease with shear displacement. Experimental evidence shows that the mean asperity contact orientation decrements in a hyperbolic fashion with fracture shear. Therefore, the evolution of asperity contact orientation distribution is modeled by assuming that the asperity contact orientation parameter, $a$, varies linearly with shear work. Based upon experimental evidence, we also observe that a critical shear displacement, $\delta_{c}$, is required before the asperity damage commences. The critical shear displacement is incorporated in the evolution laws proposed in this paper.

The derived model is used to study fracture softening and dilatation behavior under shear. The model correctly replicates the softening of fracture shear resistance, and the dependency of fracture shear strength and fracture dilatation behavior on surface roughness and fracture normal stress. Results calculated with the model are compared with experimental measurements obtained from the literature. Encouraging comparisons are obtained for: (1) shear resistance-shear displacement behavior, (2) fracture shear strength, (3) fracture dilatation behavior when sheared under constant normal stress, and (4) fracture normal stress behavior when sheared under constant fracture opening.

\section{References}

[1] Dieterich JH. Modeling of rock friction. 1. Experimental results and constitutive equations. J Geophys Res 1979;84:2161-8.

[2] Ruina AL. Slip instability and state variable friction laws. J Geophys Res 1983;88:10359-70.

[3] Barton N, Choubey V. The shear strength of rock joints in theory and practice. Rock Mech 1977;10:1-54.

[4] Bowden FP, Tabor D. The friction and lubrication of solids. Oxford, UK: Clarendon; 1950.

[5] Archard JF. Elastic deformation and the laws of friction. Proc Royal Soc London 1957;A243:190-205.

[6] Johnson KL. Contact mechanics. London, UK: Cambridge University Press; 1985.

[7] Hills DA, Nowell D, Sackfield A. Mechanics of elastic contacts. London, UK: Butterworth-Heinemann; 1992.

[8] Greenwood JA, Williamson JBP. Contact of nominally flat surfaces. Proc Royal Soc London 1966;A295:300-19.

[9] Yamada K, Takeda N, Kagami J, Naoi T. Mechanisms of elastic contact and friction between rough surfaces. Wear 1978;48:1534.

[10] Swan G. Determination of stiffness and other joint properties from roughness measurements. Rock Mech Rock Eng 1983;16:1938.

[11] Swan G, Zongqi S. Prediction of shear behavior of joints using profiles. Rock Mech Rock Eng 1985;18:183-212.

[12] Yoshioka N, Scholz CH. Elastic properties of contacting surfaces under normal and shear loads. 1. Theory. J Geophys Res 1989;94:17681-90.

[13] Boitnott GN, Biegel RL, Scholz CH, Yoshioka N, Wang W. Micromechanics of rock friction. 2. Quantitative modeling of initial friction with contact theory. J Geophys Res 1992;97:8965-78.

[14] Ford IJ. Roughness effect on friction for multi-asperity contact between surfaces. J Phys D: Appl Phys 1993;26:2219-25.

[15] Yoshioka N. A review of the micromechanical approach to the physics of contacting surfaces. Tectonophysics 1997;277:29-40.

[16] Misra A. Mechanistic model for contact between rough surfaces. J Eng Mech 1997;123(5):475-84.

[17] Misra A. Micromechanical model for anisotropic rock joints. J Geophys Res 1999;104:23175-87.

[18] Bandis S, Lumsden AC, Barton NR. Experimental studies of scale effects on the shear behavior of rock joints. Int J Rock Mech Min Sci Geomech Abstr 1981;18:1-21.

[19] Divakar MP, Fafitis A. Micromechanics based constitutive model for interface shear. J Eng Mech 1992;118(7):1317-37.

[20] Francis HA. Application of spherical indentation mechanics to reversible and irreversible contact between rough surfaces. Wear 1977;45:221-69.

[21] Brown SR, Scholz CH. Closure of random elastic surfaces in contacts. J Geophys Res 1985;90:5531-45.

[22] Nayak PR. Random process model of rough surfaces. J Lubricat Technol 1971;93:398-407.

[23] Adler RJ, Firman D. A non-Gaussian model for random surfaces. Philos Trans Royal Soc London 1981;A303:433-62.

[24] Misra A. Interfaces in particulate materials. In: Mechanics of geomaterial interfaces. The Netherlands: Elsevier Science; 1995. p. 513-36.

[25] Mindlin RD, Deresiewicz H. Elastic spheres in contact under varying oblique forces. J Appl Mech 1953;20:327-44.

[26] Wang W, Scholz CH. Wear process during frictional sliding of rock: a theoretical and experimental study. J Geophys Res 1994;99:6789-99. 
[27] Wang W, Scholz CH. Micromechanics of rock friction. 3. Quantitative modeling of base friction. J Geophys Res 1995;100: 4243-7.

[28] Biegel RL, Wang W, Scholz CH, Boitnott GN, Yoshioka N. Micromechanics of rock friction. 1. Effects of surface roughness of initial friction and slip hardening in Westerly Granite. J Geophys Res 1992;97:8951-64.

[29] Hutson RW, Dowding CH. Joint asperity degradation during cyclic shear. Int J Rock Mech Min Sci Geomech Abstr 1990;27: $109-19$. 